\title{
Avaliação da Empatia nos Médicos Residentes do Hospital Universitário Alzira Velano em Alfenas, Minas Gerais
}

\author{
Evaluation of Empathy in Medical Residents \\ of Alzira Velano University Hospital in \\ Alfenas, Minas Gerais
}

\author{
Antonio Henrique Paiva \\ Maria Natalia Andrade ${ }^{I}$ \\ Marcela Silveira Carvalho Rocha \\ José Maria Peixoto ${ }^{I !}$
}

\section{PALAVRAS-CHAVE}

- Empatia.

- Educação Médica.

- Médicos Residentes.

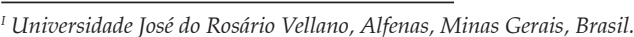

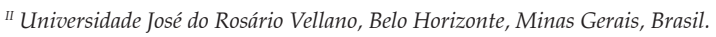




\section{KEY-WORDS}

- Empathy.

- Medical Education.

- Resident Physicians.

Recebido em 14/5/19

Aceito em 19/6/19

\section{ABSTRACT}

INTRODUCTION: Much is discussed about the importance of empathy in the patient-physician relationship. Few studies have evaluated this ability in resident physicians (RPS). OBJECTIVE: To evaluate the empathy of the resident physicians of the University Hospital Alzira Velano (HUAV), through the Jefferson Scale of Empathy (JSE), which provides 4 types of scores: General Empathy (GE); Perspective Taking (PT); Compassionate Care (CC) and Standing in Patient's Shoes (SP). METHODS: The JSE and a sociodemographic questionnaire were applied to 36 RPs of the HUAV at the beginning of the second semester of 2016, after they signed the informed consent form. RESULTS: Statistical analysis by the Mann-Whitney test showed that empathy scores were associated with variables: gender, area of performance (clinical or surgical) and period of residence in progress. For the variable gender, the following findings were observed: 1) Female RPS of the $1^{\text {st }}$ year had higher GE scores than the RPS in the $\geq 2^{\text {nd }}$ year $(p=0.01)$ and $(0.03)$, respectively; 2$)$ female $R P^{\prime} S$ of $1^{\text {st }}$ year had higher TP scores than RPS of $\geq 2$ nd year $(p=0.03)$. Regarding the area of performance, 1$)$ the GE scores of the $1^{\text {st }}$ year RPs were higher when compared to the RPs in the $\geq 2^{\text {nd }}$ year, both for the clinical $(p=0.03)$ and for the surgical area $(p=0.02) ; 2)$ RPs of the surgical area had higher PT scores in the $1^{\text {st }}$ year compared to RPS in the $\geq 2^{\text {nd }}$ year $(p=0.01)$. No association was observed between the studied factors and CC and SP scores. CONCLUSION: The data show that the empathy scores of the RPS of the final years are lower in relation to the RPS of the $1^{\text {st }}$ year, a fact also verified for female $R P$ s. Our data are in agreement with the observations of the literature and show the importance of the development of instructional strategies for the teaching of empathy among resident physicians.

\section{INTRODUÇÃO}

Evidências sugerem que a empatia é um elemento fundamental na relação médico-paciente, está associada a melhores resultados clínicos e fortalece a autonomia e a segurança do paciente $^{1}$. O conceito de empatia é multidimensional, visualizado como traço de personalidade, atributo cognitivo ou uma característica que envolve a capacidade de o médico compreender o paciente e lhe transmitir esta compreensão ${ }^{2,3}$. Tem sido tratada na literatura como possuindo dois componentes: um afetivo e outro cognitivo ${ }^{2}$. O componente afetivo é baseado em emoções, na capacidade de compreender e de partilhar os estados emocionais dos outros; já o componente cognitivo inclui a habilidade de deliberar sobre os estados emocionais de outras pessoas, diferindo do conceito de "simpatia", que é predominantemente afetivo, envolvendo sentimentos de dor e/ou de sofrimento ${ }^{4}$.

Os componentes afetivo e cognitivo da empatia fazem parte da cognição moral, que pressupõe os seguintes processos: o compartilhamento emocional, que é a capacidade de experimentar os sentimentos do outro por meio da observação; a preocupação empática, que é a motivação para cuidar de indivíduos vulneráveis; e a tomada de perspectiva, que é a capacidade de se colocar no lugar do outro e de imaginar o que está sentindo ${ }^{5}$.

A habilidade de "tomada de perspectiva" é uma das mais importantes habilidades sociocognitivas, pois por intermédio dela o indivíduo compreende que um mesmo evento pode ser visto e interpretado de várias maneiras ${ }^{6}$. Permite que um indivíduo veja os outros, interprete seus pensamentos e seja capaz de reconhecer o papel daqueles indivíduos na socieda$\mathrm{de}^{7}$. Essa habilidade é importante para o desenvolvimento do julgamento moral, que alicerça o modo como resolvemos conflitos frente a diferentes pontos de vista, como agimos e pensamos em relação aos outros ${ }^{8}$. Segundo Hojat e colaboradores ${ }^{3}$, a dimensão "tomada de perspectiva" pode ser considerada o componente-chave da empatia, referindo-se à capacidade de o profissional de saúde compreender o que experiencia, pensa e sente o paciente a partir da sua perspectiva, prestando atenção à comunicação não verbal e à linguagem corporal.

Sendo a empatia uma das habilidades fundamentais ao trabalho médico, deveria constituir uma das mais importantes habilidades a ser adquirida pelos futuros médicos durante sua formação9 . As Diretrizes Curriculares Nacionais para os cursos de graduação em Medicina elencam competências gerais 
do egresso que dependem do desenvolvimento de habilidades emocionais, entre elas a empatia ${ }^{10}$. Entretanto, estudos têm observado uma diminuição da empatia no decurso da formação do graduando de Medicina9 . Curiosamente, em alguns estudos, a empatia tende a decrescer exatamente no momento em que os estudantes começam a atender pacientes e, portanto, iniciam um contato real na atividade clínica ${ }^{11}$. Na tentativa de compreender os motivos desses achados, pesquisadores têm atribuído o declínio da empatia a diversos fatores, como problemas com a qualidade de vida dos estudantes, carga horária excessiva, contato com a doença e questões relacionadas ao enfrentamento da morte, à competitividade e ao abuso moral ${ }^{12}$.

Com objetivo de avaliar a empatia, algumas escalas foram desenvolvidas, como a Interpersonal Reactivity Index (IRI) e a Escala Jefferson de Empatia Médica - versão para estudantes (Ejem), esta última focada no contexto médico, validada para cerca de 25 idiomas e amplamente divulgada na literatura como adequada à aferição de empatia em contexto clínico ${ }^{3,13}$. A Ejem explora três fatores: tomada de perspectiva, cuidado compassivo e capacidade do profissional de se colocar no lugar do paciente ${ }^{14}$. A Ejem, validada para a língua portuguesa em um trabalho realizado na Universidade do Minho, em Portugal $^{15}$, contém 20 itens respondidos por intermédio de uma escala de Likert com variação de sete pontos $(7=$ concordo completamente a 1 = discordo completamente). Para evitar o vício de resposta, (tendência a concordar ou não com um item), dez itens da escala foram redigidos na forma afirmativa e dez itens na forma negativa. Para a obtenção do escore final, as pontuações na forma negativa devem ser invertidas antes de serem somadas ${ }^{9}$.

A Ejem avalia atributos cognitivos da empatia, tem pontuação máxima de 140 pontos $(20 \times 7)$ e fornece quatro escores: o escore de empatia geral (EG), obtido pela média da soma dos escores de todos os itens da escala; o escore de tomada de perspectiva do paciente (TP), obtido pela média da soma dos itens $2,4,5,9,10,13,15,16,17$ e 20; o escore de compaixão (CO), obtido pela média da soma dos itens 7,8,11,12,14,18 e 19; e o escore de capacidade de se colocar no lugar do outro (LO), obtido pela média da soma dos itens de 3 e 6. Sugeriu-se como tentativa de classificação dos escores da Ejem para identificar um escore baixo um valor $\leq 95$ para homens e $\leq 100$ para mulheres $^{16}$. Na versão brasileira, as propriedades psicométricas e a análise fatorial da Ejem foram adaptadas da versão portuguesa testada, e os resultados confirmaram a validade ${ }^{12}$.

A maioria dos estudos sobre empatia em medicina foi realizada no período da graduação, sendo que poucos avaliaram a empatia durante a pós-graduação. Portanto, a intensidade e como evolui essa habilidade são pouco conhecidas entre médicos residentes. Este estudo teve como objetivo avaliar os escores de empatia, por meio da Ejem, entre os MR do Hospital Universitário Alzira Velano (Huav), verificando como evoluem esses escores durante o período de formação do médico residente e identificar fatores que possam influenciar seu desenvolvimento. $\mathrm{O}$ estudo justifica-se pela necessidade de melhor compreender o desenvolvimento da empatia entre médicos residentes, para que possamos analisar suas associações, uma vez que o ambiente da residência médica pode atuar como fator estressor e contribuir para a estagnação ou mesmo para a perda da empatia.

\section{METODOLOGIA}

\section{Desenho}

Trata-se de estudo transversal, no qual a Ejem e um questionário sociodemográfico foram respondidos pelos MR do Huav no início do segundo semestre de 2016 após a leitura e assinatura do Termo de Consentimento Livre e Esclarecido (TCLE). A aplicação dos instrumentos ocorreu durante uma atividade semanal de discussão clínica. As questões foram respondidas de forma anônima.

\section{Participantes do estudo}

Foram convidados $36 \mathrm{MR}$ do Huav que se encontravam em atividade e concordaram em participar do estudo. O Huav possui programa de residência médica, com durações diferentes nas seguintes áreas: Clínica Médica (dois anos), Pediatria (dois anos), Ginecologia e Obstetrícia (três anos), Cirurgia (dois anos), Radiologia (três anos), Anestesia (três anos), Neurocirurgia (cinco anos) e Ortopedia (três anos), totalizando 36 médicos residentes. Foram excluídos os MR que se encontravam de férias, afastados e aqueles que não assinaram o TCLE.

\section{Materiais}

Para avaliação da empatia, foi utilizada a Ejem e um questionário sociodemográfico para avaliar algumas variáveis que possam se relacionar à Ejem, como: idade, especialidade em curso, se tem outra graduação na área de saúde, religião, raça, renda familiar ou pessoal e procedência.

\section{Análise de dados}

A análise dos resultados foi realizada com o software SPSS 10. Com o objetivo de comparar o Ejem entres os MR do primeiro ano (R1) e os MR dos últimos anos de residência (R2, R3 e R4) e considerando o pequeno número de residentes do terceiro e quarto ano, optamos por criar dois grupos de residentes: um formado pelos residentes do primeiro ano de residência (R1) e outro formado pelos residentes dos últimos anos de seus 
programas (R2 a R4). A análise descritiva da amostra foi realizada com base na distribuição de frequência das variáveis categóricas. Foi utilizado o teste qui-quadrado para análise de variáveis categóricas. Para avaliar a influência dos fatores ano de residência, período em curso, sexo e outros dados do questionário sociodemográfico nas médias dos escores de empatia, foi utilizada a Anova. Quando a análise indicou a influência significativa de um ou mais fatores com três categorias ou mais ou mesmo uma ou mais interações, utilizou-se o teste de comparações múltiplas de médias Least Significant Difference (LSD). Quando os pressupostos para utilização do teste $t$ de Student e/ou Anova para amostras independentes não foram aceitos e/ou a variável não apresentou distribuição normal, o teste de Mann-Whitney foi aplicado. Os resultados foram considerados significativos para uma probabilidade de significância inferior a 5\% ( $\mathrm{P} \leq 0,05)$, tendo, portanto, pelo menos 95\% de confiança nas conclusões apresentadas.

\section{RESULTADOS}

\section{Características sociodemográficas dos participantes}

A amostra foi composta por 36 médicos residentes, com idade média de 27,6 6 2,3 anos. Em relação ao período em curso, 17 residentes se encontravam no primeiro ano (R1), 11 no segundo (R2), 7 no terceiro (R3) e um no quarto ano (R4). A Tabela 1 apresenta a análise descritiva dos dados sociodemográficos da amostra.

A análise pelo teste qui-quadrado não encontrou diferença estatisticamente significativa entre os grupos de residentes R1 e R2 a R4 em relação às variáveis sexo $(p=0,50)$ e área de atuação: clínica ou cirúrgica $(\mathrm{p}=0,55)$.

Avaliação dos escores de empatia dos residentes Escores de empatia e "sexo"

A Tabela 2 apresenta as médias dos escores de EG estratificadas pelo sexo. Não há diferença significativa para os escores de EG entre os residentes do sexo masculino e feminino tanto no grupo R1 ( $p=0,31)$ quanto nos grupos R2 a R4 ( $p=0,90)$. Porém, comparando-se os escores do grupo R1 com os dos grupos R2 a R4, observa-se uma diferença significativa entre os residentes do sexo feminino: as residentes do grupo R1 apresentam escores de EG maiores que as residentes dos grupos R2 a R4.

O mesmo padrão foi observado para os escores de TP, ou seja, não foi encontrada diferença significativa entre os resi-

\begin{tabular}{|c|c|c|c|}
\hline \multicolumn{4}{|c|}{$\begin{array}{ll}\text { TABELA } 1 & \end{array}$} \\
\hline \multirow{2}{*}{$\begin{array}{l}\text { Variável } \\
\text { Sexo }\end{array}$} & \multirow[t]{2}{*}{ n (\%) } & \multirow{2}{*}{$\begin{array}{l}\text { Variável } \\
\text { Estado civil }\end{array}$} & \multirow[t]{2}{*}{ n (\%) } \\
\hline & & & \\
\hline Feminino & $19(52,8)$ & Casado/relação estável & $7(19,4)$ \\
\hline Masculino & $17(47,2)$ & Solteiro & $29(80,6)$ \\
\hline \multicolumn{2}{|l|}{ Área da residência médica? } & \multicolumn{2}{|l|}{ Raça } \\
\hline Clínica Médica & $4(11,1)$ & Branca & $32(88,9)$ \\
\hline Pediatria & $7(19,5)$ & Parda & $3(8,30)$ \\
\hline Ginecologia e Obstetrícia & $3(8,3)$ & Amarela & $1(2,8)$ \\
\hline Radiologia & $2(5,6)$ & & \\
\hline Clínica Cirúrgica & $5(13,9)$ & & \\
\hline Anestesia & $8(22,2)$ & & \\
\hline Neurocirurgia & $4(11,1)$ & Renda familiar estimada & \\
\hline Ortopedia & $3(8,3)$ & $<5$ salários mínimos & $6(17,1)$ \\
\hline \multicolumn{2}{|l|}{ Motivo da área escolhida? } & 5 a 20 salários mínimos & $23(65,7)$ \\
\hline Econômica & $1(2,9)$ & > 20 salários mínimos & $6(17,1)$ \\
\hline Vocacional & $26(76,5)$ & Doença crônica pessoal? & \\
\hline Área a que mais se adapta & $7(20,6)$ & Sim & $5(13,9)$ \\
\hline \multicolumn{2}{|l|}{ Regular no curso? } & Não & $31(86,1)$ \\
\hline Sim & $36(100)$ & Doença crônica familiar? & \\
\hline Não & $0(0)$ & Sim & $19(52,8)$ \\
\hline \multicolumn{2}{|l|}{ Outra graduação em saúde? } & Não & $17(47,2)$ \\
\hline Sim & $3(8,3)$ & Acredita em Deus? & \\
\hline \multirow[t]{2}{*}{ Não } & $33(91,7)$ & Sim & $36(100)$ \\
\hline & & Não & $0(0)$ \\
\hline
\end{tabular}

Base de dados: 36 residentes; $n=$ número de residentes; $\%=$ percentual 
dentes do sexo masculino e feminino tanto no grupo R1 ( $\mathrm{p}=$ $0,42)$ como nos grupos R2 a R4 ( $p=0,90)$. Porém, observa-se diferença significativa para os residentes do sexo feminino entre os grupos, sendo que as residentes R1 apresentam escores de TP significativamente maiores do que os das residentes R2 a R4 (Tabela 3).

Para os escores $\mathrm{CO}$, nenhuma diferença estatisticamente significativa foi encontrada entre os residentes do sexo masculino e feminino no grupo $\mathrm{R} 1(\mathrm{p}=0,67)$ e nos grupos $\mathrm{R} 2 \mathrm{a}$ $\mathrm{R} 4$ ( $\mathrm{p}=0,60)$. Não se observou diferença significativa ao comparar os escores de $\mathrm{CO}$ dos residentes do grupo $\mathrm{R} 1$ aos dos residentes dos grupos R2 a R4 em relação tanto ao sexo masculino $(\mathrm{p}=0,42)$ como ao sexo feminino ( $\mathrm{p}=0,55)$. Também não foi encontrada diferença significativa para os escores LO entre os residentes do sexo masculino e feminino no grupo
$\mathrm{R} 1(\mathrm{p}=0,47)$ e nos grupos R2 a $\mathrm{R} 4(\mathrm{p}=0,60)$. E não foi encontrada diferença significativa entre os escores dos grupos R1 e R2 a R4 em relação ao sexo masculino $(p=0,96)$ ou feminino $(\mathrm{p}=0,21)$.

Escores de empatia e "área de atuação" (clinica ou cirúrgica) Quanto à área de atuação, não houve diferença estatisticamente significativa entre os residentes que escolheram área cirúrgica e os que optaram pela área clínica no que se refere ao escore de EG, tanto para o grupo R1 ( $p=0,67)$, quanto para os grupos R2 a R4 ( $\mathrm{p}=0,42)$. Porém, o grupo de residentes R1 apresentou escores de EG significativamente maiores do que os dos residentes R2 a R4 em ambas as áreas de atuação (Tabela 4).

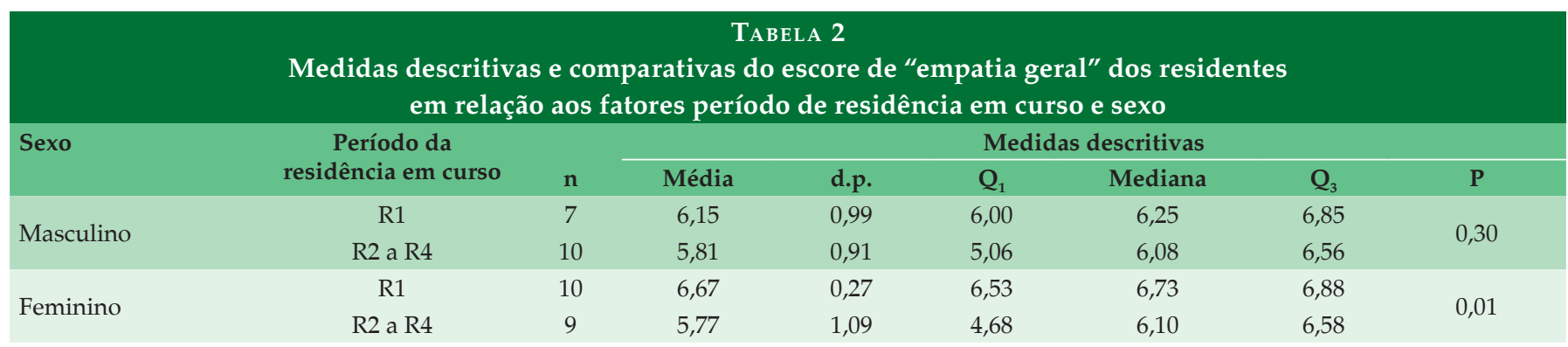

Nota: Base de dados: 36 residentes; $p=$ teste de Mann-Whitney.

\begin{tabular}{|c|c|c|c|c|c|c|c|c|}
\hline \multicolumn{9}{|c|}{$\begin{array}{l}\text { Medidas descritivas e comparativas do escore de "tomada de perspectiva do doente" } \\
\text { pelos residentes em relação aos fatores residência em curso e sexo }\end{array}$} \\
\hline \multirow[t]{2}{*}{ Sexo } & \multirow{2}{*}{$\begin{array}{l}\text { Período da } \\
\text { residência em curso }\end{array}$} & \multirow[b]{2}{*}{$\mathbf{n}$} & \multicolumn{6}{|c|}{ Medidas descritivas } \\
\hline & & & Média & d.p. & $\mathrm{Q}_{1}$ & Mediana & $\mathrm{Q}_{3}$ & $\mathbf{P}$ \\
\hline \multirow{2}{*}{ Masculino } & R1 & 7 & 6,43 & 0,56 & 5,80 & 6,60 & 7,00 & \multirow{2}{*}{0,31} \\
\hline & R2 a R4 & 10 & 5,84 & 1,05 & 4,95 & 6,15 & 6,65 & \\
\hline \multirow{2}{*}{ Feminino } & $\mathrm{R} 1$ & 10 & 6,75 & 0,21 & 6,58 & 6,75 & 6,93 & \multirow{2}{*}{0,03} \\
\hline & $\mathrm{R} 2 \mathrm{a} \mathrm{R} 4$ & 9 & 6,00 & 0,92 & 5,60 & 6,10 & 6,70 & \\
\hline
\end{tabular}

Nota: Base de dados: 36 residentes; $p=$ teste de Mann-Whitney.

\begin{tabular}{|c|c|c|c|c|c|c|c|c|}
\hline \multirow{3}{*}{$\begin{array}{c}\text { Área de atuação } \\
\text { escolhida }\end{array}$} & \multicolumn{8}{|c|}{$\begin{array}{l}\text { TАВЕLA } 4 \\
\text { Medidas descritivas e comparativas do escore de "empatia geral" dos residentes em } \\
\text { relação aos fatores período da residência em curso e área de atuação médica }\end{array}$} \\
\hline & \multirow{2}{*}{$\begin{array}{c}\text { Período da } \\
\text { residência em curso }\end{array}$} & \multirow[b]{2}{*}{$\mathbf{n}$} & \multicolumn{6}{|c|}{ Medidas descritivas } \\
\hline & & & Média & d.p. & $Q_{1}$ & Mediana & $\mathrm{Q}_{3}$ & $\mathbf{P}$ \\
\hline \multirow{2}{*}{ Cirurgia } & R1 & 10 & 6,33 & 0,87 & 6,04 & 6,73 & 6,85 & \multirow{2}{*}{0,02} \\
\hline & $\mathrm{R} 2 \mathrm{a} \mathrm{R} 4$ & 13 & 5,60 & 1,09 & 4,33 & 5,95 & 6,58 & \\
\hline \multirow{2}{*}{ Clínica } & $\mathrm{R} 1$ & 7 & 6,64 & 0,28 & 6,30 & 6,65 & 6,95 & \multirow{2}{*}{0,03} \\
\hline & $\mathrm{R} 2$ a $\mathrm{R} 4$ & 6 & 6,20 & 0,46 & 5,91 & 6,30 & 6,56 & \\
\hline
\end{tabular}

Nota: Base de dados: 36 residentes; $p=$ teste de Mann-Whitney. 
Quanto ao escore TP, o resultado mostra que não existe diferença estatística entre os residentes da área cirúrgica e os da área clínica, tanto no grupo $\mathrm{R} 1(\mathrm{p}=0,67)$ como nos grupos R2 a R4 ( $p=0,13)$. Observou-se diferença significativa entre os residentes da cirurgia em relação ao período em curso, sendo que os residentes R1 apresentaram escores maiores do que os residentes R2 a R4 (Tabela 5).

Para os escores CO, não foi verificada diferença estatisticamente significativa entre os residentes e área de atuação médica em curso (clínica e cirúrgica), tanto no grupo R1 (p $=0,74)$ como nos grupos $\mathrm{R} 2$ a $\mathrm{R} 4(\mathrm{p}=0,64)$. O escore de CO também não apresentou diferença entre os grupos de residentes R1 e R2 a R4 tanto na área de atuação cirúrgica $(p=0,45)$ como na clínica $(p=0,37)$. Em relação aos escores LO, nenhuma diferença significativa foi identificada entre os grupos de residentes e a área de atuação (clínica e cirúrgica), tanto no grupo R1 ( $p=0,60)$ como nos grupos R2 a R4 ( $p=0,10)$. O escore LO também não demonstrou diferença entre os grupos de residentes $\mathrm{R} 1$ e R2 a R4 na área de atuação: cirurgia $(p=0,65)$ e clínica $(p=0,30)$.

\section{DISCUSSÃO}

Este estudo teve como objetivo avaliar os escores de empatia, por meio da Ejem, entre os MR do Huav, procurando identificar fatores que possam influenciá-los. Dos fatores sociodemográficos estudados, os únicos que se relacionaram aos escores da Ejem e apresentaram diferenças significativas foram: sexo, área de atuação (clínica ou cirúrgica) e período em curso.

\section{Escores da Ejem versus gênero e período da residência em} curso

O presente trabalho não encontrou diferença para os escores de EG e TP entre os MR do sexo masculino e feminino tanto nos anos iniciais, como nos anos finais do curso. Observou-se que esses escores eram maiores entre as mulheres nos anos iniciais da residência em relação àquelas que se encontravam do segundo ano de residência em diante. Hojat e Gonella ${ }^{16}$, estudando 2.637 alunos com número de participantes semelhante em relação a gênero e utilizando a Ejem, observaram escore de empatia maior no gênero feminino. De forma geral, nesse estudo, as mulheres obtiveram escores mais elevados do que os dos homens. Contudo, esse achado não é unânime. Estudo realizado em Roma, utilizando a Ejem, com 289 médicos de diferentes especialidades e em várias fases da carreira não mostrou diferenças significativas entre os gêneros, a especialidade e a fase da carreira ${ }^{17}$. Tem-se verificado entre as mulheres maior capacidade de identificar, compreender e expressar estados que envolvem emotividade ${ }^{18}$. A diferença da empatia nos diferentes gêneros tem sido relatada por diversos autores, sendo explicada mais como uma diminuição da empatia nas mulheres e não como o aumento desta nos homens ${ }^{19,20}$.

Semelhante fato foi observado neste estudo, em que não houve predominância significativa da empatia nem entre os gêneros nem entre especialidades. A atenuação da empatia pode ser explicada pela influência de fatores negativos, tais como falta de modelo, aumento progressivo de conteúdo técnico a ser ensinado, ambiente hostil de trabalho, medo de cometer erros, currículo exigente e pouco sono ${ }^{9}$. Barnet e colaboradores ${ }^{21}$ citam o impacto emocional diante de situações estressantes que é maior nas mulheres por estas apresentarem maior vulnerabilidade emocional ${ }^{21}$. Moreto $^{22}$, ao comparar empatia entre homens e mulheres utilizando a Ejem e a escala de reatividade multidimensional interpessoal (Emri), observou que estas possuem escore de empatia maior, mas não identificou diferença entre homens e mulheres na dimensão cognitiva da escala ${ }^{22}$

\section{Escores da Ejem versus área de atuação e período da residência em curso}

Este estudo não encontrou diferença para os escores de EG e TP entre os MR que optaram por uma área clínica ou cirúrgica para a amostra total. Observou-se que os escores de EG dos MR nos anos iniciais eram maiores que os encontrados nos MR do segundo ano em diante, independentemente da área

\begin{tabular}{|c|c|c|c|c|c|c|c|c|}
\hline \multicolumn{9}{|c|}{$\begin{array}{l}\text { Medidas descritivas e comparativas do escore de "tomada de perspectiva do doente" pelos } \\
\text { residentes em relação aos fatores período da residência em curso e área de atuação médica }\end{array}$} \\
\hline \multirow{2}{*}{$\begin{array}{l}\text { Área de atuação } \\
\text { escolhida }\end{array}$} & \multirow{2}{*}{$\begin{array}{l}\text { Período da } \\
\text { residência em curso }\end{array}$} & \multirow[b]{2}{*}{$\mathbf{n}$} & \multicolumn{6}{|c|}{ Medidas descritivas } \\
\hline & & & Média & d.p. & $\mathrm{Q}_{1}$ & Mediana & $\mathbf{Q}_{3}$ & $\mathbf{P}$ \\
\hline \multirow{2}{*}{ Cirurgia } & R1 & 10 & 6,59 & 0,40 & 6,33 & 6,70 & 6,93 & \multirow{2}{*}{0,01} \\
\hline & R2 a R4 & 13 & 5,69 & 1,03 & 4,70 & 6,10 & 6,50 & \\
\hline \multirow{2}{*}{ Clínica } & $\mathrm{R} 1$ & 7 & 6,66 & 0,45 & 6,60 & 6,70 & 7,00 & \multirow{2}{*}{0,53} \\
\hline & $\mathrm{R} 2$ a $\mathrm{R} 4$ & 6 & 6,40 & 0,64 & 5,70 & 6,70 & 6,85 & \\
\hline
\end{tabular}

Nota: Base de dados: 36 residentes; $p=$ teste de Mann-Whitney. 
de atuação. Para os escores de TP, entre os MR da área cirúrgica, foi observado que os escores dos MR dos anos iniciais eram maiores que os escores dos MR do segundo ano em diante. Muitos estudos têm mostrado uma diminuição significativa nos escores de empatia com o passar dos anos de graduação, com poucos trabalhos avaliando a empatia por área de atuação em médicos residentes ${ }^{23}$. Bailey ${ }^{24}$ mostrou que os estudantes de Medicina que pretendiam exercer uma área clínica obtiveram escore de empatia maior que aqueles que planejavam atuar em áreas cirúrgicas. Estudos realizados com médicos apresentaram o mesmo padrão que o observado com os estudantes. Num estudo realizado com 704 médicos, utilizando a Ejem, os envolvidos com a área clínica obtiveram escores mais elevados que aqueles das áreas cirúrgicas e de imagem ${ }^{25}$. Foi observado que estagiários do último ano que preferem a prática geral como especialidade apresentaram atitudes mais empáticas que os estagiários da área cirúrgica ${ }^{26}$.

Neste estudo, não foi observada diferença significativa entre os residentes da área clínica e cirúrgica no que se refere ao escore de empatia geral, mas houve uma diminuição significativa deste escore com o passar dos anos de residência nas duas áreas. Essa diminuição está de acordo com o que foi observado literatura. $\mathrm{O}$ declínio da empatia no decorrer dos anos de formação pode ser atribuído a diversos fatores, como problemas com a qualidade de vida dos residentes, carga horária excessiva, contato com a doença, questões relacionadas ao enfrentamento da morte, competitividade e abuso moral ${ }^{27}$. Outros fatores que podem contribuir para a erosão da empatia durante o curso são falta de exemplo por parte dos preceptores e grande quantidade de informação técnica a ser transmitida, o que diminui o tempo para a abordagem de questões humanísticas que delineiam a relação médico-paciente ${ }^{28}$.

\section{ESCORES DE CO E LO}

Não foi observada nenhuma relação dos escores de CO e LO quanto aos fatores estudados: sexo, área de atuação escolhida e período da residência em curso. Paro e colaboradores ${ }^{12}$ mostraram que os escores $\mathrm{CO}$ e LO surgiram na análise de componentes principais na versão brasileira da Ejem como primeiro e segundo da escala respectivamente, diferentemente da escala original, em que a TP foi o principal escore. Esses autores lembram que no Brasil o cristianismo é a religião predominante, e características como compaixão e caridade são consideradas fundamentais ${ }^{12}$. Se a compaixão é entendida como uma sobreposição entre cognição e emoção ${ }^{18}$, pode-se inferir que as emoções desempenham um papel importante nas habilidades humanísticas no cuidado com o paciente entre os estudantes e médicos brasileiros, assim como o fator LO, que pode refletir a importância da compaixão entre estes. Esses fatores são intrínsecos nestes profissionais, sem a necessidade de treinamento e sem grande influência do contexto em que estão inseridos ${ }^{12}$.

O componente TP foi o terceiro escore na versão brasileira da JSPE e o primeiro na escala original. As diferenças culturais podem explicar parcialmente esses resultados, mas as questões curriculares devem ser levadas em consideração ${ }^{12}$. Esse fator está relacionado a habilidades cognitivas: processamento de informações, raciocínio, avaliação e empatia de comunicação ${ }^{18}$. Não existe, na maioria das escolas médicas brasileiras, treinamento para essa habilidade, que ocorre em momentos isolados no currículo médico ${ }^{27}$. Isto sugere erroneamente que a empatia, de modo geral, é uma característica interna ou uma disposição natural, em vez de uma competência a ser ensinada, treinada e avaliada. A ausência de oportunidades para a prática da empatia poderia explicar a diminuição deste escore, observado neste trabalho ${ }^{29}$, principalmente entre o gênero feminino, pela maior sensibilidade em sofrer influência do contexto, e entre a equipe cirúrgica, pelo maior contato com situações estressantes ${ }^{9}$.

\section{LIMITAÇÕES DO ESTUDO}

Ressaltamos que o questionário utilizado, de autoavaliação, pode não refletir o comportamento real dos participantes, mas, sim, uma resposta socialmente correta. É o fenômeno da desejabilidade social citado por Edwards ${ }^{30}$, em que o entrevistado, de forma inconsciente, tende a responder às questões não como pensa, sente ou age, mas como são reconhecidas como socialmente aceitáveis. A explicação de que as respostas são confidenciais pode reduzir esta tendência, mas pode ser insuficiente. A pequena amostra e a possibilidade de R2 a $\mathrm{R} 4$ estarem em momentos diferentes podem ter interferido no resultado.

\section{CONCLUSÃO}

Nosso estudo demonstrou, numa amostra com 36 médicos residentes, que os escores de EG e TP foram semelhantes entre os residentes das áreas clínicas e cirúrgicas, e maiores entre os residentes do primeiro ano em relação aos do segundo ano em diante. Observou-se, ainda, que residentes do sexo feminino dos últimos anos de residência apresentaram escores menores em relação às médicas residentes do primeiro ano. Nenhuma associação aos fatores estudados foi observada para os escores CO e LO. Nossos dados concordam com observações da literatura e podem contribuir para a discussão da necessidade do desenvolvimento de estratégias instrucionais para o ensino da empatia entre médicos residentes. 


\section{REFERÊNCIAS}

1. Loureiro J, Gonçalves-Pereira M, Trancas B, Caldas-de-Almeida JM, Castro-Caldas A. Empatia na relação médico-doente. Acta MedPort 2011; 24(S2):431-442.

2. Hemmerdinger JM, Stoddart SDR, Lilford RJ. A.Systematic review of tests of empathy in medicine. BMC Medical Education 2007; 7(24):1-8. DOI:10.1186/1472-6920-7-24

3. Hojat, M, Mangione S, Nasca TJ, Cohen MJM, Gonnella JS, Erdmann JB, et al. The Jefferson Scale of Physician Empathy: development and preliminary psychometric data. Educational and Psychological Measurement 2001; 61(2):349-365.

4. Hojat M. Empathy in Patient Care: Antecedentes, Development, Measurement and outcomes. New York: Springer, 2007

5. Decety J, Cowell JM.The complex relation between morality and empathy. Trends Cognitive Sciences 2014; 18(7):337339. DOI: $10.1016 /$ j.tics.2014.04.008.

6. Moll H, Meltzoff AN. How Does It Look? Level 2 Perspective-taking at 36 Months of Age. Child Development 2011; 82(2):661-673.

7. Kohlberg L. Moral stages and moralization: The cognitive-developmental approach. In T. Lickona (Ed.), Moral development and behavior: Theory, research and social issues. New York: Holt, Rinehart and Winston, 1976

8. Selman RL. Social-cognitive understanding: a guide to educational and clinical practice. In T. Lickona (Ed.), Moral development and behavior. New York: Holt, Rinehart and Winston, 1976

9. Hojat M, Vergare M, Maxwell K, Brainard G, Herrine S, Veloski JI, et al. The devil is in the third year a longitudinal study of erosion of empathy in medical school. Academic Medicine 2009;84(9):1182-1191. DOI: 10.1097/ ACM.0b013e3181b17e55.

10. Arora S, Ashrafian H, Davis R, Athanasiou T, Darzi A, Sevdalis N. Emotional intelligence in medicine: a systematic review through the context of the ACGME competencies. Medical Education 2010;44 (8):749-64. DOI: 10.1111/j.1365-2923.2010.03709.x.

11. Provenzano BC, Machado APG, Rangel MTAS, Aranha RN. A empatia médica e a graduação em medicina. Revista Hospital Universitário Pedro Ernesto 2014;13(4):19-25. DOI:10.12957/rhupe.2014.13941

12. HBMS, Daud-Gallotti RM, Tibério IC, Pinto RMC, Martins MA. Brazilian version of the Jefferson Scale of Empathy: psychometric properties and factor analysis. BMC Medical Education 2012; 12:73. DOI:10.1186/1472-6920-12-73
13. Costa FD, Azevedo RCS.Empatia, relação médico-paciente e formação em medicina: um olhar qualitativo.Revista Brasileira de Educação Médica 2010;34(2):261-269.

14. Hojat M, Lanoue M.Exploration and confirmation of the latent variable structure of the Jefferson scale of empathy. International Journal of Medical Education 2014; 5:73-81. DOI: 10.5116/ijme.533f.0c41.

15. Magalhães E, Dechamplain A, Salgueira A, Costa MJ. Empatia Médica: Adaptação e validação de uma escala para estudantes de medicina. Actas do VII Simpósio Nacional de Investigação em Psicologia Universidade do Minho; 2010 fev. 4-6, Portugal:2010

16. Hojat M, Gonnella JS. Eleven years of data on the Jefferson Scale of Empathy-Medical Student Version (JSE-S): Proxy norm data and tentative cutoff scores. Medical Principles and Practice 2015; 24(4):344-350. DOI:10.1159/000381954

17. Di Lillo M, Cicchetti A, Loscalzo A, Taroni F, Hojat M. The Jefferson Scale of Physician Emphaty: Preliminary Psycometrics and Group Comparisons in Italian Physicians. Acad Med. 2009;84 (9):1198-202. DOI: 10.1097/ ACM.0b013e3181b17b3f.

18. Hojat M. Empathy in Patient Care. Antecedents, Development, Measurement, and Outcomes. New York: Springer, 2007.

19. Arnold RM, Martin SC, Parker RM. Taking care of patients-does it matter whether the physician is a woman? Western F Med 1988; 149:729-33.

20. Dufort F, Maheux B. When female medical students are the majority: do numbers really make a difference. Journal of the American Medical Women's Association 1995;50(1):4-6

21. Barnett RC, Biener L, Baruch GK. Gender and Stress. New York: Free Press, 1987

22. Moreto G. Avaliação da empatia de estudantes de medicina em uma universidade na cidade de São Paulo utilizando dois instrumentos. São Paulo; 2015. Doutorado [Tese] - Universidade de São Paulo. DOI:10.11606/T.5.2015.tde19062015-154448.

23. Hojat M, Mangione S, Nasca TJ, Rattner S, Erdmann JB, Gonnella JS, et al. An empirical study of decline in empathy in medical school. Medical Education 2004;38(9):934-941.

24. Bailey BA. Empathy in Medical Students: Assessment and Relationship to speciality choice. Virginia; 2001. PhD [Thesis] - University of Virginia.

25. Hojat M, Gonnella JS, Nasca TJ, Mangione S, Vergare M, Magee M. Physician empathy: Definition, components, measurement and relationship to gender and specialty. Am J Psychiatry. 2002; 159(9):1563-1569 
26. Batenburg V, Smal JA, Lodder A, Melker RA. Are Professional attitudes related to gender and medical specialty? Medical Education 1999; 33:489-492.

27. Neumann M, Edelhäuser F, Tauschel D, Fischer MR, Wirtz M, Woopen C, et al. Empathy decline and its reasons: a systematic review of studies with medical students and residents. Academic Medicine 2011;86(8):996-1009.

28. Hogan R. Development of an empathy scale. Journal of Consulting and Clinical Psychology 1969;33(3):307-316

29. Tempski $P$, Martins MA, Paro HB. Teaching and learning resilience: a new agenda in medical education. Medical Education 2012;46(4): 345-346.DOI:10.1111/j.13652923.2011.04207.x.

30. Edwards AL. The social desirability variable in personality assessment and research. New York: Dryden Press, 1957.

\section{CONTRIBUIÇÃO DOS AUTORES}

O autor principal foi responsável pela elaboração do projeto de pesquisa, revisão bibliográfica, coleta e análise dos dados, discussão dos resultados e a produção do manuscrito. Os demais autores contribuíram, auxiliando o autor principal em todas as etapas da pesquisa, bem como na produção e revisão do manuscrito.

\section{CONFLITO DE INTERESSES}

Os autores declaram não haver conflito de interesses.

\section{ENDEREÇO PARA CORRESPONDÊNCIA}

Antônio Henrique de Paiva

Rua Geraldo Freitas da Costa, 120 - Bairro Cruz Preta

Alfenas-Minas Gerais

CEP 37130-000.

E-mail: antonio.paiva@unifenas.br 